\title{
Stretching Solves a Mystery of Magic-Angle Graphene
}

\author{
Numerical simulations show that discrepancies between experiments on \\ graphene bilayers can be attributed to tiny amounts of strain applied to \\ the samples.
}

By Marric Stephens

S tacking two sheets of graphene and rotating one relative to the other by a certain "magic" angle induces in the system a rich array of phases, including superconductivity (see Viewpoint: Graphene Reveals its Strange Side). Experimentalists have been mapping these phases since 2018, when the idea of "twisted bilayer graphene" (TBG) was first introduced. But where some groups have found an insulating phase, others have observed a semimetal. Now, using numerical simulations, Daniel Parker at Harvard University and colleagues have identified strain as the crucial factor that could explain these different outcomes [1].

In magic-angle TBG, the lattices of two graphene sheets are offset by about $1.1^{\circ}$. This misalignment produces a moiré pattern that repeats over a length scale much longer than that of the graphene's crystal structure. (The unit cell of graphene is just a few carbon atoms, while that of TBG encompasses thousands.) This crystalline complexity represents a challenge

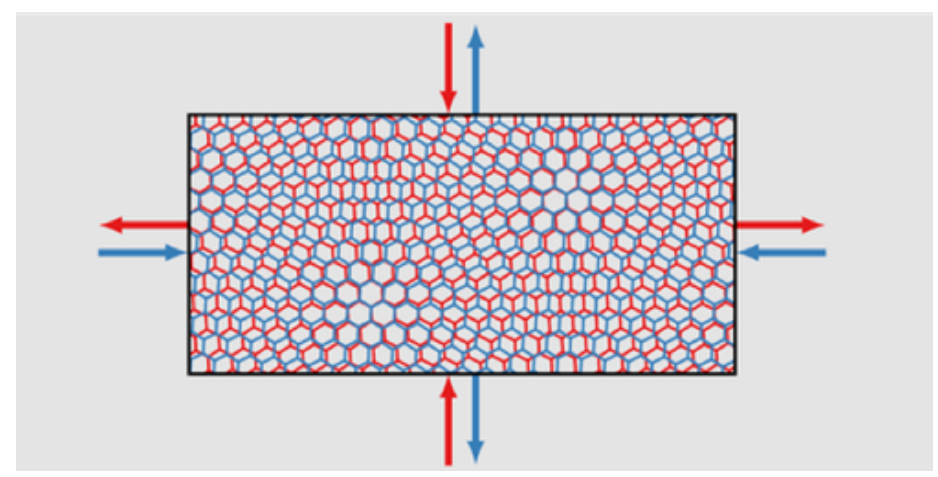

Credit: D. E. Parker, T. Soejima, J. Hauschild, M. P. Zalatel, and N. Bultinck for researchers studying the material using numerical methods.

Parker and his colleagues avoided this problem by simulating a small number of low-energy electronic bands around the material's Fermi level and "freezing" electrons with energies outside of this range. Simulating only this subset allowed them to model a sufficiently large TBG system to reveal the material's peculiar properties.

The results of their simulations indicate that the phase transition from an insulator to a semimetal can be driven by applying a heterostrain of $0.1-0.2 \%$ to the graphene sheets. This small strain falls within the range expected for experiments. The researchers say that eliminating the strain from experiments will be tricky, but if it can be done, strain could eventually represent an important "tuning knob" for the material.

Marric Stephens is a Corresponding Editor for Physics based in Bristol, UK.

\section{REFERENCES}

1. D. E. Parker et al., "Strain-induced quantum phase transitions in magic-angle graphene,” Phys. Rev. Lett. 127, 027601 (2021). 